\title{
Successful neoadjuvant peptide receptor radionuclide therapy for an inoperable pancreatic neuroendocrine tumour
}

\author{
Tiago Nunes da Silva', (Loes) M L F van Velthuysen², Casper H J van Eijck³, \\ Jaap J Teunissen ${ }^{4}$, (Hans) J Hofland ${ }^{1}$ and Wouter W de Herder ${ }^{1}$ \\ 1Departments of Internal Medicine, ENETS Centre of Excellence, ${ }^{2}$ Pathology, ${ }^{3}$ Surgery, and ${ }^{4}$ Radiology \& Nuclear \\ Medicine, Erasmus MC, University Medical Centre, Rotterdam, the Netherlands
}

Correspondence
should be addressed
to T N da Silva
Email
tiago_fcml@yahoo.com

\section{Summary}

Non-functional pancreatic neuroendocrine tumours (NETs) can present with advanced local or distant (metastatic) disease limiting the possibility of surgical cure. Several treatment options have been used in experimental neoadjuvant settings to improve the outcomes in such cases. Peptide receptor radionuclide therapy (PPRT) using beta emitting radiolabelled somatostatin analogues has been used in progressive pancreatic NETs. We report a 55-year-old female patient with a $12.8 \mathrm{~cm}$ pancreatic NET with significant local stomach and superior mesenteric vein compression and liver metastases. The patient underwent treatment with [177Lutetium-DOTA ${ }^{0}, \mathrm{Tyr}^{3}$ ] octreotate (177Lu-octreotate) for the treatment of local and metastatic symptomatic disease. Six months after 4 cycles of 177 lutetium-octreotate, resolution of the abdominal complaints was associated with a significant reduction in tumour size and the tumour was rendered operable. Histology of the tumour showed a $90 \%$ necrotic tumour with abundant hyalinized fibrosis and haemorrhage compatible with PPRT-induced radiation effects on tumour cells. This report supports that PPRT has a role in unresectable and metastatic pancreatic NET.

\section{Learning points:}

- PRRT with ${ }^{177} \mathrm{Lu}$-octreotate can be considered a useful therapy for symptomatic somatostatin receptor-positive pancreatic NET.

- The clinical benefits of PRRT with ${ }^{177} \mathrm{Lu}$-octreotate can be seen in the first months while tumour reduction can be seen up to a year after treatment.

- PRRT with ${ }^{177} \mathrm{Lu}$-octreotate was clinically well tolerated and did not interfere with the subsequent surgical procedure.

- PRRT with ${ }^{177} \mathrm{Lu}$-octreotate can result in significant tumour reduction and may improve surgical outcomes. As such, this therapy can be considered as a neoadjuvant therapy.

\section{Background}

Pancreatic neuroendocrine tumours (NET) presenting with metastatic disease (stage IV disease) are an important clinical challenge with a 5-year survival rate of approximately 35\% (1). Although surgical treatment is considered the only curative option for localized pancreatic NETs, this is not usually the case for patients presenting with locally advanced or metastatic disease. In these patients, a significant local mass effect 
with arterial or venous invasion and significant liver metastasis can render stage IV patients inoperable (2). Neoadjuvant treatments for advanced NET patients are considered experimental, but in a few selected cases, they have been shown to be successful for stabilizing the disease or even allowing down-staging of both the primary tumour and its metastasis (3). Although PPRT is considered a second- or third-line therapy for progressive somatostatin receptor (SSTR)-positive pancreatic NETs (1), it has been recently shown to be an effective and safe neoadjuvant treatment option in a few case reports and small case series of advanced inoperable pancreatic NET patients $(4,5)$.

We report on a patient with an advanced symptomatic and inoperable stage IV pancreatic NET with lymph node and liver metastases. In this case, treatment with ${ }^{177} \mathrm{Lu}$-octreotate induced not only resolution of symptoms, but also a surprising macroscopic disappearance of hepatic and lymph node metastasis and allowed successful R0 surgery.

\section{Case presentation}

We report on a 55-year-old female patient who was evaluated in her local hospital for an 18-month clinical history of severe abdominal pain, nausea, vomiting and weight loss. She had no complaints of secretory diarrhoea or complaints suggestive of high or low blood glucose. On physical examination, a palpable epigastric mass was detected and further diagnostic work-up led to a diagnosis of a grade 2 , stage IV metastatic pancreatic NET.

\section{Investigation}

Blood analysis revealed haemoglobin of $8.2 \mathrm{mmol} / \mathrm{L}$ $(13.2 \mathrm{~g} / \mathrm{dL})$, leucocytes of $5.510^{9} / \mathrm{L}$ and thrombocytes of $142 \times 10^{9} / \mathrm{L}$, a creatinine level of $68 \mu \mathrm{mol} / \mathrm{L}$, normal liver enzymes, normal blood glucose and normal potassium levels and an elevated chromogranin A of $2050 \mu \mathrm{g} / \mathrm{L}$ (reference value $(\mathrm{RV})<94 \mu \mathrm{g} / \mathrm{L}$ ).

Abdominal CT showed a $12.8 \mathrm{~cm}$ mass in the pancreatic body with compression of the stomach and the superior mesenteric vein a $37 \mathrm{~mm}$ lymph node metastasis in the gastrohepatic ligament and several multilobar liver metastases (Fig. 1A). An 111indiumpentetreotide scintigram showed pathological uptake in the pancreatic tumour, pathologic lymph node and liver lesions as described on CT (Fig. 2A). Biopsy showed a well-differentiated grade 2 neuroendocrine tumour (Ki-67 index of 3\%) with positive immunostaining for chromogranin, synaptophysin and the somatostatin receptor subtype $2 \mathrm{a}$ (SSTR2A) receptor.

A multidisciplinary tumour board proposed the patient for PPRT treatment with ${ }^{177} \mathrm{Lu}$-octreotate for control of the symptomatic advanced disease.

\section{Treatment}

The patient underwent 4 treatment cycles with ${ }^{177} \mathrm{Lu}$-octreotate between January and August 2015 after 111indium-pentetreotide scintigram showed a Krenning scale grade 3 uptake (higher than normal liver), with a cumulative administered activity of $30.6 \mathrm{GBq}$. The treatment had no major side effects. While the first 24-h post therapy scan after the first cycle of ${ }^{177} \mathrm{Lu}$-octreotate showed an accumulation of ${ }^{177} \mathrm{Lu}$-octreotate in accordance with baseline ${ }^{111}$ Indium-octreotide scintigraphy, the following 3 treatment cycles showed a progressive lower accumulation ${ }^{177} \mathrm{Lu}$-octrotate in the known pathological lesions suggesting a reduction in tumour mass. After the second treatment cycle, the patient already reported a significant improvement in abdominal complaints and after the third cycle, a volume reduction in the pancreatic lesion was observed. At the end of the 4 therapy cycles, the patient again reported a significant decrease in her abdominal complaints, improved quality

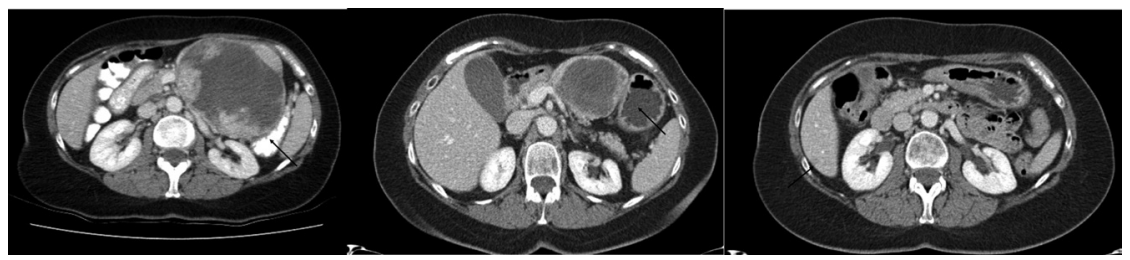

Figure 1

Abdominal CT before PPRT (A) and after PPRT (B) and after surgery (C). Abdominal CT showing a $12.8 \mathrm{~cm}$ pancreatic NET with stomach compression (arrow). Abdominal CT 6 months after PPRT showing a $5 \mathrm{~cm}$ reduction in the primary pancreatic NET size, with significant decompression of the stomach cavity (arrow). 


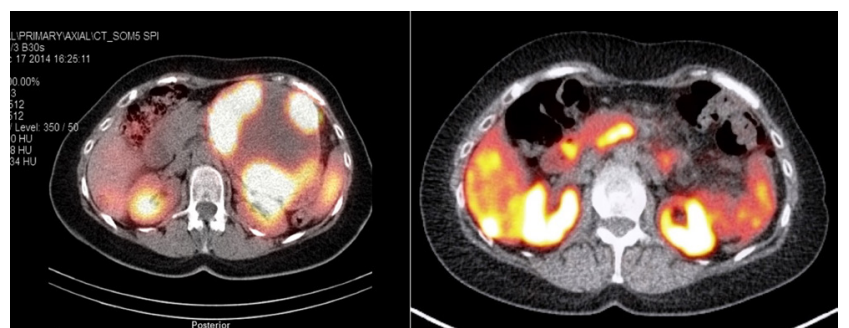

Figure 2

Initial diagnostic ${ }^{111}$ indium-octreotide scintigraphy SPECT-CT (A) showing pathological uptake of the radioligand in the pancreatic tumour and a ${ }^{68}$ Gallium DOTATE-PET-CT (B) showing complete disappearance of the pancreatic lesion after surgery.

of life and weight loss stoppage (an increase from 69 to $71.7 \mathrm{~kg}$ was observed). Laboratory showed a decrease in chromogranin A level from 2050 to $97 \mu \mathrm{g} / \mathrm{L}(\mathrm{RV}<94 \mu \mathrm{g} / \mathrm{L})$ without anaemia.

\section{Outcome and follow-up}

Abdominal CT performed 2 months after the last treatment cycle of ${ }^{177} \mathrm{Lu}$-Octreotate showed a decrease in the solid component of the pancreatic body tumour from 12.8 to $9.0 \mathrm{~cm}$, with no visible compression of the superior mesenteric vein, a disappearance of the $37 \mathrm{~mm}$ lymph node metastasis and a significant decrease in size of the pre-existing liver metastases. A subsequent CT scan 6 months after the last treatment cycle of ${ }^{177} \mathrm{Lu}$-octreotate showed a further decrease in the pancreatic body mass (from 9.0 to $7.8 \mathrm{~cm}$ ) and disappearance of most of the liver metastases (Fig. 1B). The plasma chromogranin A levels was $30 \mu \mathrm{g} / \mathrm{L}(\mathrm{RV}<94 \mu \mathrm{g} / \mathrm{L})$. At this stage, a distal pancreatectomy plus splenectomy was performed. No major surgical complications were observed. Histopathology showed a well-differentiated NET of the pancreas with positive immunohistochemistry for synaptophysin, chromogranin, SSTR2A receptor and a Ki-67 index of 1\%. Significant PRRT-related radiation effects were observed with necrosis in over $90 \%$ of the tumour and abundant hyalinization, sclerosis and haemorrhagic areas (Fig. 3B). Two out of 10 lymph nodes were found to have nodal metastasis with extensive hyalinization and sclerosis.

Five months after surgery, ${ }^{68}$ gallium-octreotate positron emission tomography-CT (PET-CT) showed disappearance of the pancreatic and lymph node lesions and only tracer accumulation in a focal $9 \mathrm{~mm}$ liver lesion (Fig. 2B). Subsequently, this pancreatic NET liver metastasis was surgically completely removed and histologically confirmed. The patient was tumour-free one year after wedge resection of the liver. This was exactly 3 years after the start of PRRT with ${ }^{177} \mathrm{Lu}$-octreotate.

\section{Discussion}

Pancreatic NETs are uncommon tumours and a significant proportion of patients presents with locally advanced disease (compression or invasion of adjacent structures, including blood vessels) and or metastatic disease at diagnosis (6).

Locally advanced disease may exclude the patient from the only potential curative treatment (R0 excision) (7). In this context, several locally advanced pNET are deemed unresectable because patients who undergo resection with a macroscopic positive margin have a similar overall survival as those who do not undergo resection. The risk of macroscopic positive margin increases with increasing tumour contact to the major arteries or veins such as a greater than 180 degrees encasement of the superior mesenteric artery (SMA), any celiac artery abutment, unreconstructable superior mesenteric vein (SMV)/portal
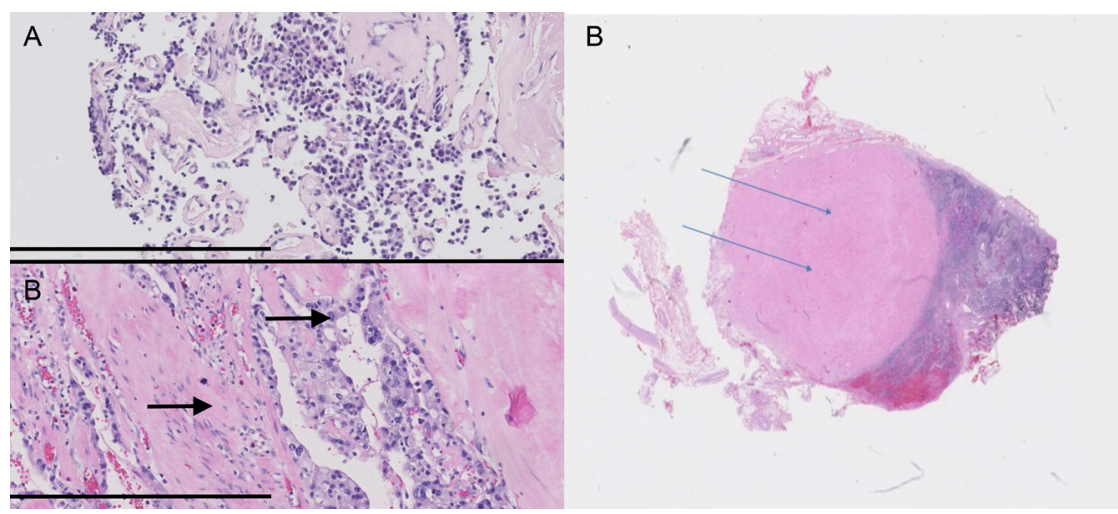

\section{Figure 3}

Haematoxylin and eosin (HE) staining showing the difference in the cellular component between the biopsy (A) (before PPRT with ${ }^{177} \mathrm{Lu}$-octreotate) and the surgical specimen (B) (after PPRT) of the pancreatic NET. Micrographs with similar magnification $\mathrm{I}-\mathrm{I}=400 \mu \mathrm{m}$. The arrows highlight the reduction in the cellular component and the increase in both the nuclear and cytological size after PPRT treatment. This haematoxylin and eosin (HE) staining of the pancreatic lymph node resection specimen (C) shows the extensive 177Lu-octreotate treatment effect: significant hyalinization and sclerosis of the lymph node metastasis is seen (arrows) with no residual tumour cells left. 
vein (PV) occlusion or aortic invasion or encasement. However, in selected patients with large pNETs, and in centres with appropriate surgical experience, aggressive surgery for locally advanced tumours can be considered, when the benefit-risk ratio is considered favourable (8). In patients with both local and metastatic disease, surgical resection of primary tumour was associated with an increase in both cancer-specific survival and overall survival (7).

Randomized controlled studies on the best sequential order of treatments for locally advanced pNETs are lacking (9). On one hand based on previous encouraging experience with pancreatic adenocarcinomas, neoadjuvant therapy followed by surgical treatment in responders has been tried as a therapeutic option in locally advanced pancreatic NET patients. Several therapeutic options have been described mainly in case reports or small case series. These include biological agents (sunitinib or everolimus), systemic chemotherapy, arterial embolization, chemoembolization, radiofrequency ablative therapy, cytoreductive surgery and PPRT. Recently, a retrospective positive experience has been reported on neoadjuvant PPRT-induced tumour downstaging (successful surgical R0/R1 surgery in 31\% of cases without major adverse effects) (5). On the other hand, a sequential approach of surgery followed by PPRT has been proposed as having a radiobiological rationale (surgical debulking would reduce the tumour burden allowing higher radiation concentrations to smaller lesions). This approach has shown on a nonrandomized prospective study, a progression-free survival but not an overall survival advantage (10). Considering the lack of highquality evidence at present, the best sequential approach to local and metastatic pNET remains a matter of debate and as such, each case should be discussed on a regular basis in a multidisciplinary tumour board.

In the reported case, PRRT with ${ }^{177} \mathrm{Lu}$-octreotate was well tolerated and not only lead to resolution of the severe abdominal pain but also increased the odds for successful surgery. In fact, this treatment should, therefore, be considered as neoadjuvant PRRT. As previously described, ${ }^{177} \mathrm{Lu}$-octreotate PRRT did not negatively impact subsequent surgery despite its radiation effects being clearly apparent in the tumour tissue: extensive tumour necrosis associated with abundant hyalinization, sclerosis and tissue haemorrhage (Fig. 3C). These histological results have only been documented in a few cases of successful PPRT-induced tumour regression (5). Although symptomatic relive occurred shortly after ${ }^{177} \mathrm{Lu}$-octreotate
PRRT, progressive tumour regression, due to radiation damage, was observed 6 months after the last treatment cycle compared to the 2-month evaluation. As such, the timing for surgery should take into account the ongoing tumour shrinkage, which has been consistently described up to one year after the last treatment cycle (10).

In conclusion, we report the use of ${ }^{177} \mathrm{Lu}$-octreotate as a useful neoadjuvant therapy in a locally advanced somatostatin receptor subtype-positive pancreatic NET, although this therapy was initially only considered for tumour control.

\section{Declaration of interest}

The authors declare that there is no conflict of interest that could be perceived as prejudicing the impartiality of the research reported.

\section{Funding}

This research did not receive any specific grant from any funding agency in the public, commercial or not-for-profit sector.

\section{Patient consent}

The hospital's medical ethics committee approved the study of peptide receptor radiotherapy with ${ }^{177} \mathrm{Lu}$-octreotate. The patient gave written informed consent for participation in this study.

\section{Author contribution statement}

Tiago Nunes da Silva gathered the data and wrote the text after obtaining permission from Prof. de Herder. M L F van Velthuysen reviewed the pathological slides. Casper $\mathrm{H} J$ van Eijck reviewed the manuscript. Jaap J Teunissen reviewed the manuscript and performed PPRT treatment. J Hofland and Wouter de Herder followed the patient and revised the manuscript.

\section{References}

1 Scarpa A, Mantovani W, Capelli P, Beghelli S, Boninsegna L, Bettini R, Panzuto F, Pederzoli P, delle Fave G \& Falconi M. Pancreatic endocrine tumors: improved TNM staging and histopathological grading permit a clinically efficient prognostic stratification of patients. Modern Pathology 201023 824-833. (https://doi. org/10.1038/modpathol.2010.58)

2 Pavel M, O'Toole D, Costa F, Capdevila J, Gross D, Kianmanesh R, Krenning E, Knigge U, Salazar R, Pape UF, et al. ENETS consensus guidelines update for the management of distant metastatic disease of intestinal, pancreatic, bronchial Neuroendocrine Neoplasms (NEN) and NEN of unknown primary site. Neuroendocrinology 2016 103 172-185. (https://doi.org/10.1159/000443167)

3 Perysinakis I, Aggeli C, Kaltsas G \& Zografos GN. Neoadjuvant therapy for advanced pancreatic neuroendocrine tumors: an emerging treatment modality? Hormones 201615 15-22. (https://doi. org/10.14310/horm.2002.1636)

4 Kwekkeboom DJ, de Herder WW, Kam BL, van Eijck CH, van Essen M, Kooij PP, Feelders RA, van Aken MO \& Krenning EP. 
Treatment with the radiolabeled somatostatin analog [177 Lu-DOTA 0,Tyr3] octreotate: toxicity, efficacy, and survival. Journal of Clinical Oncology 200826 2124-2130. (https://doi.org/10.1200/ JCO.2007.15.2553)

5 van Vliet EI, van Eijck CH, de Krijger RR, Nieveen van Dijkum EJ, Teunissen JJ, Kam BL, de Herder WW, Feelders RA, Bonsing BA, Brabander T, et al. Neoadjuvant treatment of nonfunctioning pancreatic neuroendocrine tumors with [177Lu-DOTA0,Tyr3] octreotate. Journal of Nuclear Medicine 201556 1647-1653. (https:// doi.org/10.2967/jnumed.115.158899)

6 Pavel M, Baudin E, Couvelard A, Krenning E, Öberg K, Steinmüller T, Anlauf M, Wiedenmann B, Salazar R \& Barcelona Consensus Conference participants. ENETS consensus guidelines for the management of patients with liver and other distant metastases from neuroendocrine neoplasms of foregut, midgut, hindgut, and unknown primary. Neuroendocrinology 201295 157-176. (https://doi org/10.1159/000335597)

7 Lianyuan T, Dianrong X, Sadula A, Ye C, Chen Q, Wang H, Zhang Z, Zhang L, Tao M \& Yuan C. Surgical resection of primary tumor improves survival of pancreatic neuroendocrine tumor with liver metastases. Oncotarget 201745 79785-79792. (https://doi. org/10.18632/oncotarget.19523)

8 Norton JA, Harris EJ, Chen Y, Visser BC, Poultsides GA, Kunz PC, Fisher GA \& Jensen RT. Pancreatic endocrine tumors with major vascular abutment, involvement, or encasement and indication for resection. Archives of Surgery 2011146 724-732. (https://doi. org/10.1001/archsurg.2011.129)

9 Bertani E, Fazio N, Radice D, Zardini C, Grana C, Bodei L, Funicelli L, Ferrari C, Spada F, Partelli S, et al. Resection of the primary tumor followed by peptide receptor radionuclide therapy as upfront strategy for the treatment of G1-G2 pancreatic neuroendocrine tumors with unresectable liver metastases. Annals of Surgical Oncology 201623 981-999. (https://doi.org/10.1245/s10434-016-5550-3)

10 Kwekkeboom DJ, Kam BL, van Essen M, Teunissen JJ, van Eijck CH, Valkema R, de Jong M, de Herder WW \& Krenning EP. Somatostatin receptor-based imaging and therapy of gastroenteropancreatic neuroendocrine tumors. Endocrine-Related Cancer 201017 R53-R73. (https://doi.org/10.1677/ERC-09-0078)

Received in final form 12 March 2018

Accepted 21 March 2018 